\title{
A Whitening Filtering Approach for the Detection of Abnormal Intra-QRS Potentials in Signal-Averaged Electrocardiograms
}

\author{
Chun-Cheng Lin
}

\begin{abstract}
The detection of extremely low-amplitude and high-frequency abnormal intra-QRS potentials (AIQP) in signal-averaged electrocardiograms is a potential technique to improve the diagnostic performance of the risk of ventricular arrhythmias. Instead of using the modeling residuals of the previous studies, this study develops a whitening filtering approach to whiten the input QRS wave and use the optimized filter coefficients to detect the presence of AIQP due to the high-frequency characteristics of AIQP that may increase the higher order coefficients. To demonstrate the performance of the proposed method for detecting the AIQP, a color noise with a frequency band ranged from $40 \mathrm{~Hz}$ to $250 \mathrm{~Hz}$ is embedded into the normal QRS wave to simulate AIQP, and the detection performance of $A I Q P$ is tested under four signal-to-noise ratios (SNRs) (AIQP vs. QRS) including -50 dB, -46 dB, - $40 \mathrm{~dB}$, and $\mathbf{- 3 4} \mathrm{dB}$. The study results demonstrate that the presence of AIQP can increase the amplitude of the higher order coefficients of the whitening filter. The accuracy of AIQP detection can at least reach $75 \%, 83 \%, 92 \%$, and $96 \%$ under SNRs of $-50 \mathrm{~dB},-46 \mathrm{~dB},-40 \mathrm{~dB}$, and $-34 \mathrm{~dB}$, respectively, in the $\mathrm{X}, \mathrm{Y}$ and $\mathrm{Z}$ leads.
\end{abstract}

Index Terms-Abnormal Intra-QRS potentials, signal-averaged electrocardiogram, ventricular arrhythmias, whitening filtering.

\section{INTRODUCTION}

The presence of ventricular late potentials (VLP) [1]-[6] or abnormal intra-QRS potentials (AIQP) in a signal-averaged electrocardiogram (SAECG) has been proved to be related with the development of sustained ventricular tachycardia (VT) [7]-[12]. The VLP detection focuses on the evaluation of low-amplitude and high-frequency components at the terminal QRS complex, and has excellent negative predictive accuracy for stratifying the risk of ventricular arrhythmias in patients who are recovering from myocardial infarction, and for identifying patients with ischemic heart disease and unexplained syncope, who are likely to have inducible sustained ventricular tachycardia [5], [6]. However, since VLP overlap the end of the large-amplitude QRS complex, an extremely poor signal-to-noise ratio (SNR) or noise interference can limit VLP detection. Its positive predictive accuracy is not yet sufficiently high to justify interventions for those whose

Manuscript received April 10, 2013; revised July 11, 2013. This work was supported by the National Science Council of the Republic of China, Taiwan under Contract no. 101-2320-B-167-001-.

Chun-Cheng Lin is with the Department of Electrical Engineering, National Chin-Yi University of Technology, No.57, Sec. 2, Jhongshan Rd., Taiping Dist., Taichung 41170, Taiwan (e-mail: cclin@ ncut.edu.tw). analysis results are abnormal.

The AIQP can be considered as another version of VLP, which distribute over the entire QRS interval, and represent the low-amplitude and high-frequency notches and slurs. Several previous studies have demonstrated that the combination of VLP and AIQP parameters can improve the diagnostic performance of SAECG for the evaluation of the risk of ventricular arrhythmias [7]-[12]. Gomis et al. [7], [8] developed an autoregressive moving average (ARMA) model built in the discrete cosine transform (DCT) domain to estimate the normal QRS components and analyze the AIQP by the modeling residuals. However, the current ARMA parametric modeling is in the DCT domain (i.e. frequency domain) for estimating the AIQP hence the transient and unpredictable features in the time domain may not be accurately extracted. Instead of extracting the waveform of AIQP, our previous studies [10]-[12] proposed the analysis of unpredictable intra-QRS potentials (UIQP) based on a prediction model to detect the signals with sudden slope change within the QRS wave. The main limitation of the current methods for the detection of AIQP is that the AIQP is estimated by the modeling residual signal which may be directly affected by the background noise. Another limitation is that the optimal model order is unknown and is mainly dependent on clinical data. These limitations reduce the clinical feasibility of AIQP analysis.

In order to improve the limitations of the current parametric and prediction models, this study develops a new approach for the detection of AIQP based on a whitening filtering technology to whiten the input QRS wave and use the optimized filter coefficients to detect the presence of AIQP instead of using the modeling residual signal of the current methods.

\section{MATERIALS AND METHODS}

\section{A. Materials and Data Acquisition}

Five study groups consisting of one normal and four AIQP groups were recruited to test the performance of the proposed whitening filtering approach for the detection of AIQP. The normal group consisted of 42 normal Taiwanese. Because all the normal subjects had a normal clinical history, physical examination, and 12-lead ECG and echocardiogram, they had a low possibility of presenting AIQP in the SAECG. The four AIQP groups were constructed under four SNRs (AIQP vs. the normal QRS) of $-50 \mathrm{~dB}(1: 300),-46 \mathrm{~dB}(1: 200),-40$ $\mathrm{dB}(1: 100)$, and $-34 \mathrm{~dB}(1: 50)$, respectively, by adding a color noise with a frequency band ranged from $40 \mathrm{~Hz}$ to 250 
$\mathrm{Hz}$ into the QRS waves of the normal subjects to simulate the presence of different AIQP magnitudes. The color noise was produced by a four-order Butterworth filter with a pass band ranged from $40 \mathrm{~Hz}$ to $250 \mathrm{~Hz}$ and with a normally distributed white noise input.

The high-resolution electrocardiograms were recorded at rest in a supine position using a commercially available Simens-Elema Megacart ${ }^{\circledR}$ machine. A bipolar, orthogonal X, $\mathrm{Y}$ and $\mathrm{Z}$ lead system was used. A sample of 10 minutes raw ECG with 12-bit resolution at $2 \mathrm{kHz}$ was stored on computer hard disk for subsequent analysis. Signal averaging was performed to lower the effects of the random noise. The final noise level of SAECG was less than $0.7 \mu \mathrm{V}$ measured by a four-order bidirectional Butterworth filter with a pass band ranged from $40 \mathrm{~Hz}$ to $250 \mathrm{~Hz}$. The starting point (onset) and end point (offset) of the QRS wave were obtained from the analysis of the vector magnitude [5], [6].

\section{B. Whitening Filtering}

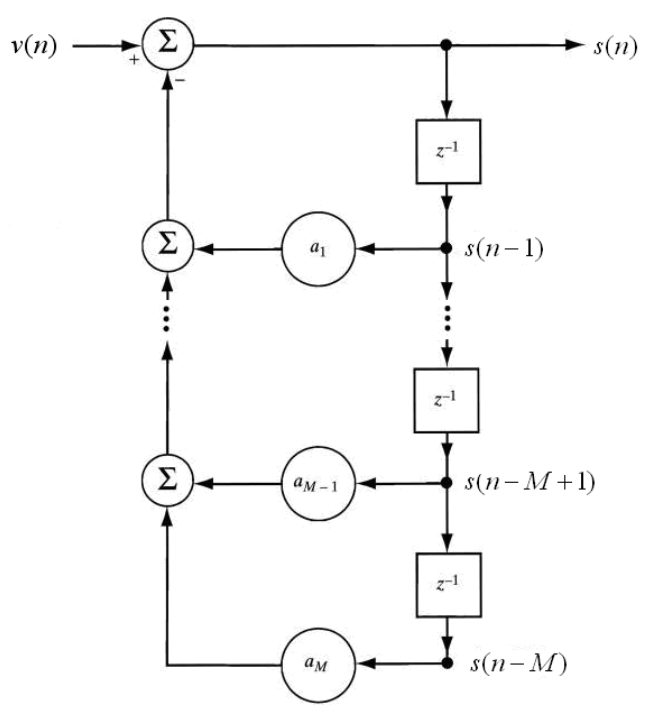

Fig. 1. Block diagram of an Mth-order AR model.

The detection of AIQP based on a whitening filter assumes that the QRS wave, including the normal QRS wave and the possible AIQP, is an autoregressive (AR) random process which can be produced by an AR model with a normally distributed white noise input [13], [14]. Therefore, if the QRS wave is filtered with a whitening filter defined by the inverse of the AR model, it can be whitened as a white noise process. Under this assumption, the coefficients of the AR model or whitening filter would represent the full waveform characteristics of the QRS wave. Hence this paper tries to estimate the optimized whitening filter coefficients for the input QRS, and then uses these coefficients to analyze the normal QRS wave and the possible AIQP. It is expected that the normal QRS wave with large amplitude would be concentrated at the lower order coefficients of the whitening filter, and the low-amplitude and high-frequency AIQP would distribute over the higher order coefficients.

Fig. 1 shows the block diagram of an $M$ th-order AR model, where the input signal $v(n)$ is a white noise process with a flat power spectral density, and the AR model parameters are denoted as $a_{1}, a_{2}, \cdots, a_{M}$ [14]. The output signal $s(n)$ can be represented as

$$
s(n)+a_{1} s(n-1)+\cdots+a_{M} s(n-M)=v(n)
$$

or

$$
s(n)=-a_{1} u(n-1)-\cdots-a_{M} s(n-M)+v(n)
$$

To solve the AR model parameters $\left\{a_{1}, a_{2}, \cdots, a_{M}\right\}$, both sides of (1) are multiplied by $s(n-l)$ to obtain

$$
E\left[\sum_{k=0}^{M} a_{k} s(n-k) s(n-l)\right]=E[v(n) s(n-l)]
$$

Assume that the white noise $v(n)$ is uncorrelated with the shifted input signal $s(n-l), l>0$; hence, the expectation value on the right side of (3) is equal to 0 . Furthermore, if we interchange the expectation and summation operators on the left side of (3), we can obtain

$$
\sum_{k=0}^{M} a_{k} r(l-k)=0, l>0
$$

where $a_{0}=1$, and $r(l-k)=E[s(n-k) s(n-l)]$ is the autocorrelation function of the output signal of the AR model. We can further express (4) as the following difference equation:

$$
\begin{aligned}
r(l) & =-a_{1} r(l-1)-a_{2} r(l-2)-\cdots-a_{M} r(l-M) \\
& =w_{1} r(l-1)+w_{2} r(l-2)+\cdots+w_{M} r(l-M)
\end{aligned}
$$

where $w_{k}=-a_{k}, k=1,2, \cdots, M$. Let $l=1,2, \cdots, M$, and the $M$ simultaneous equations can be derived as follows:

$$
\left[\begin{array}{cccc}
r(0) & r(1) & \cdots & r(M-1) \\
r(1) & r(0) & \cdots & r(M-2) \\
\vdots & \vdots & \ddots & \vdots \\
r(M-1) & r(M-2) & \cdots & r(0)
\end{array}\right]\left[\begin{array}{c}
w_{1} \\
w_{2} \\
\vdots \\
w_{M}
\end{array}\right]=\left[\begin{array}{c}
r(1) \\
r(2) \\
\vdots \\
r(M)
\end{array}\right]
$$

The matrix form can be expressed as follows:

$$
\mathbf{R} \mathbf{w}=\mathbf{r}
$$

where $\mathbf{R}$ is the autocorrelation matrix formed by the output signal, $\mathbf{w}=\left[w_{1}, w_{2}, \cdots, w_{M}\right]^{T}$ is the vector formed by the AR model parameters, and $\mathbf{r}$ is the autocorrelation vector formed by the output signal. We can get the AR model parameters $a_{k}=-w_{k}, k=1,2, \cdots, M$ by solving (7). This study introduces General Levinson Recursion to recursively solve the equations which are a set of Hermitian Toeplitz equations of the form given in (7). In addition, let $l=0$, and the expectation value of the right side of (3) can be expressed as follows:

$$
E[v(n) s(n)]=E[v(n) v(n)]=\sigma_{v}^{2}
$$

where $\sigma_{v}^{2}$ is the power or variance of the input white noise. From (3) and (8), we can obtain

$$
\sigma_{v}^{2}=\sum_{k=0}^{M} a_{k} r(k)
$$

Hence if the autocorrelation values of $r(0), r(1), \ldots$, and 
$r(M)$ are given, the variance of the white noise can be determined by (9). The root-mean-square (RMS) value of the AR model or whitening filter coefficients is further used to define the AIQP parameter for the AIQP detection as follows:

$$
\operatorname{AIQP}(m, M)=\sqrt{\frac{1}{M} \sum_{i=m}^{M} w_{i}^{2}}
$$

where $M$ is the model order, $m$ is the starting point of the coefficient interval for calculating the AIQP parameter, $1 \leq m \leq M$.

\section{Statistical Analysis}

All statistical analyses were undertaken using the Statistical Package for the Social Sciences ${ }^{\circledR}$. Normal distribution tests were performed on all quantitative variables. Statistical significance was defined as a $p$ value less than 0.05 . Comparisons between pairwise groups were performed using a Student $t$ test for normally distributed continuous variables. Levene's test was used to check the homogeneity of variance between variables. The Mann Whitney $U$ and Wilcoxon Rank Sum tests were used for those non-normally distributed variables [15].

TABLE I: SUMMARY RESULTS OF THE AIQP DETECTION

\begin{tabular}{|c|c|c|}
\hline Groups & $\begin{array}{l}\text { AIQP Parameters } \\
\text { (mean } \pm \text { STD) }\end{array}$ & $\begin{array}{c}\text { Accuracy } \\
(\%)\end{array}$ \\
\hline \multicolumn{3}{|c|}{ Lead X } \\
\hline Normal & $0.045 \pm 0.036$ & \\
\hline$-50 \mathrm{~dB}$ AIQP & $0.052 \pm 0.039$ & 82.2 \\
\hline$-46 \mathrm{~dB}$ AIQP & $0.058 \pm 0.042$ & 89.9 \\
\hline$-40 \mathrm{~dB}$ AIQP & $0.074 \pm 0.049$ & 95.5 \\
\hline$-34 \mathrm{~dB}$ AIQP & $0.097 \pm 0.054$ & 96.6 \\
\hline \multicolumn{3}{|c|}{ Lead Y } \\
\hline Normal & $0.038 \pm 0.030$ & \\
\hline$-50 \mathrm{~dB}$ AIQP & $0.041 \pm 0.031$ & 75.4 \\
\hline-46 dB AIQP & $0.044 \pm 0.032$ & 83.6 \\
\hline$-40 \mathrm{~dB}$ AIQP & $0.054 \pm 0.035$ & 92.5 \\
\hline-34 dB AIQP & $0.071 \pm 0.039$ & 96.4 \\
\hline \multicolumn{3}{|c|}{ Lead Z } \\
\hline Normal & $0.030 \pm 0.024$ & \\
\hline$-50 \mathrm{~dB}$ AIQP & $0.034 \pm 0.026$ & 78.4 \\
\hline$-46 \mathrm{~dB}$ AIQP & $0.038 \pm 0.029$ & 86.6 \\
\hline$-40 \mathrm{~dB}$ AIQP & $0.049 \pm 0.035$ & 94.8 \\
\hline$-34 \mathrm{~dB}$ AIQP & $0.068 \pm 0.045$ & 98.2 \\
\hline
\end{tabular}

STD: standard deviation.

\section{RESULTS}

Because the true waveform and randomness of AIQP are unknown, this study introduces a color noise with a frequency band ranged from $40 \mathrm{~Hz}$ to $250 \mathrm{~Hz}$, which is same with the one used in the standard time-domain analysis for the detection of VLP [5], [6], to simulate AIQP. Fig. 2 illustrates an X lead QRS wave from a normal subject simulating the normal QRS wave (top), and a normally distributed white noise simulating the AIQP (bottom). The SNR (AIQP vs. the normal QRS) is $-34 \mathrm{~dB}$ (1:50). Fig. 3 compares the absolute amplitude of the estimated whitening filter coefficients of the normal QRS wave not adding AIQP (solid line with dot markers) and adding AIQP (solid line with square markers), using a whitening filter of order $M=50$. The RMS values of the two QRS waves are adjusted to be equal. It is obvious that most of the higher order coefficients of the normal QRS wave adding AIQP have larger amplitudes in comparison with that of the normal QRS wave not adding AIQP. In order to quantify the effects induced by the presence of AIQP, this study defined the AIQP parameter by the RMS value of filter coefficients. If the starting point of the coefficient interval for calculating the AIQP parameter is set at $m=11$, the AIQP parameter of the normal QRS wave adding AIQP is 0.0606 , which is larger than 0.0204 of the normal QRS wave not adding AIQP. Hence the presence of AIQP can cause increased amplitudes in the higher order coefficients of the whitening filter.

Table I further shows the results of the AIQP detection for the normal and AIQP groups in the leads $\mathrm{X}, \mathrm{Y}$ and $\mathrm{Z}$ using a whitening filter of the order $M=50$. The starting point of the parameter interval for calculating the AIQP parameter was set at $m=11$. The groups named $-50 \mathrm{~dB}$ AIQP, $-46 \mathrm{~dB}$ AIQP, -40 dB AIQP and -34 dB AIQP were constructed by adding color noises into the QRS waves of the normal subjects using the SNRs of $-50 \mathrm{~dB},-46 \mathrm{~dB},-40 \mathrm{~dB}$ and -34 $\mathrm{dB}$, respectively. The AIQP parameter for each QRS wave in the AIQP groups was estimated by ensemble averaging over 100 independent experiments. All of the mean values of AIQP parameters in the four AIQP groups were significantly larger than those of the normal group in the $\mathrm{X}, \mathrm{Y}$ and $\mathrm{Z}$ leads $(p<0.05)$. The accuracy was further defined to evaluate the performance of the proposed method for the detection of AIQP as follows:

$$
\text { Accuracy }(\%)=\left(1-\frac{\text { No. of false detections }}{\text { No. of all AIQP detections }}\right) \times 100 \%
$$

where the false detection represents the result that the estimated AIQP parameter of a normal QRS wave not adding the simulated AIQP is larger than that of a normal QRS wave after adding AIQP. The number of all AIQP detections is 4,200 times for 42 study subjects in each lead. For example, the number of false detections in the group of $-34 \mathrm{~dB}$ AIQP for lead $\mathrm{X}$ is 143 , hence the accuracy is $96.6 \%$. The study results shown in Table 1 demonstrate that the accuracy of AIQP detection can at least reach 75\%, 83\%, 92\%, and 96\% under the SNRs of $-50 \mathrm{~dB},-46 \mathrm{~dB},-40 \mathrm{~dB}$, and $-34 \mathrm{~dB}$, respectively, in the $\mathrm{X}, \mathrm{Y}$ and $\mathrm{Z}$ leads.

\section{DisCUSSION AND CONCLUSIONS}

This study proposes a new whitening filtering approach to detect the low-amplitude, high-frequency and random AIQP embedded in the large-amplitude QRS wave. The input QRS wave is assumed as an AR process that can be whitened by a 
whitening filter. Hence the coefficients of the whitening filter can dominate all of the characteristics of the QRS wave including possible AIQP. Because most of the energy of the large-amplitude normal QRS wave is concentrated at the lower frequency band, it can be characterized by the lower order coefficients. However the AIQP is expected to be distributed over the higher order coefficients due to its high-frequency feature. Hence if the AIQP appear in the QRS wave, the whitening filter should have increased amplitudes in the higher order coefficients. The study results can confirm this viewpoint and illustrate that the presence of the AIQP can increase most of the amplitudes of the higher order coefficients of the whitening filter. This study further defined an AIQP parameter based on the RMS value of the whitening filter coefficients to quantify the effects of AIQP. Different AIQP levels were adopted to test the performance of the proposed whitening filtering approach. The study results demonstrate that all of the mean values of AIQP parameters of the QRS waves with AIQP were significantly larger than those of the normal QRS wave without AIQP in the X, Y and $\mathrm{Z}$ leads. However the AIQP under an extremely low SNR may not be detected correctly because the AIQP may be mixed with part of the normal QRS wave. As shown in Table 1 , the accuracy is only $82.8 \%, 75.4 \%$ and $78.4 \%$ under -50 $\mathrm{dB}$ SNR in the leads $\mathrm{X}, \mathrm{Y}$ and $\mathrm{Z}$, respectively. If the SNR reaches $-34 \mathrm{~dB}$, the accuracy is higher than $96 \%$ in all leads.

The main difference between the proposed whitening filtering approach and the previous studies [7]-[12] using the parametric model and prediction model is that the proposed approach used the filter coefficients to detect the AIQP, while the previous methods used the modeling or prediction errors to evaluate AIQP. The modeling or prediction errors not only include the AIQP but also the background noise hence the accuracy of AIQP detection would be limited by the presence of the background noise. If the background noise is a random process, it would not be whitened by the whitening filtering and remained in the filter output. Hence the proposed method would not be sensitive to the background noise. Furthermore, the proposed approach does not need to decide the optimal model order for the detection of AIQP because a high order filter is required for the whitening filtering. The filter order of $M=50$ and the starting point of the coefficient interval for calculating the AIQP parameter, $m=11$, are determined empirically in this study. However a high filter order may have some problems caused by overfitting which would decrease the accuracy of the AIQP detection. The proposed method is based on the assumption that the features of the normal QRS wave are concentrated at the lower order coefficients of the whitening filter. However, if this assumption does not hold, the accuracy of AIQP detection would be reduced because both the AIQP and part of the normal QRS wave may present in the higher order coefficients simultaneously.

\section{ACKNOWLEDGMENT}

The author would like to thank the National Science Council of the Republic of China, Taiwan, for financially supporting this research under Contract no. 101-2320-B-167-001-. Staff of the Hemodialysis Unit and patients of the Cardiology Department at Jen-Chi General
Hospital are appreciated for their kind assistance and cooperation in this investigation.

\section{REFERENCES}

[1] M. B. Simson, "Use of signals in the terminal QRS complex to identify patients with ventricular tachycardia after myocardial infarction," Circulation, vol. 64, no. 2, pp. 235-242, Aug. 1981.

[2] J. R. Jarrett and N. C. Flowers, "Signal-averaged electrocardiography: history, techniques, and clinical applications," Clin. Cardiol., vol. 14, no. 12, pp. 984-994, Dec. 1991.

[3] P. Lewandowski, O. Meste, R. Maniewski, T. Mroczka, K. Steinbach, and H. Rix, "Risk evaluation of ventricular tachycardia using wavelet transform irregularity of the high-resolution electrocardiogram," Med. Biol. Eng. Comput., vol. 38, no. 6, pp. 666-673, Nov. 2000.

[4] R. Vazquez, E. B. Caref, F. Torres, M. Reina, J. A. Guerrero, and N El-Sherif, "Reproducibility of time-domain and three different frequency-domain techniques for the analysis of the signal-averaged electrocardiogram," J. Electrocardiol., vol. 33, no. 2, pp. 99-105, Apr. 2000.

[5] G. Breithardt, M. E. Cain, N. El-Sherif, N. Flowers, V. Hombach, M. Janse, M. B. Simson, and G. Steinbeck, "Standards for analysis of ventricular late potentials using high-resolution or signal-averaged electrocardiography: a statement by a task force committee of the European Society of Cardiology, the American Heart Association, and the American College of Cardiology," J. Am. Coll. Cardiol., vol. 17, no. 5, pp. 999-1006, Apr. 1991.

[6] M. E. Cain, J. L. Anderson, M. F. Arnsdorf, J. W. Mason, M. M. Scheinman, and A. L. Waldo, "Signal-averaged electrocardiography," $J$. Am. Coll. Cardiol., vol. 27, no. pp. 238-249, Jan. 1996.

[7] P. Gomis, D. L. Jones, P. Caminal, E. J. Berbari, and P. Lander, "Analysis of abnormal signals within the QRS complex of the high-resolution electrocardiogram," IEEE Trans. Biomed. Eng., vol. 44, no. 8, pp. 681-693, Aug., 1997

[8] P. Lander, P. Gomis, R. Goyal, E. J. Berbari, P. Caminal, R. Lazzara and J. S. Steinberg, "Analysis of abnormal intra-QRS potentials Improved predictive value for arrhythmic events with the signal-averaged electrocardiogram," Circulation, vol. 95, no. 6, pp. 1386- 1393, Mar. 1997.

[9] E. J. Berbari, E. A. Bock, A. C. Cházaro, X. Sun, and L. Sörnmo, "High-resolution analysis of ambulatory electrocardiograms to detect possible mechanisms of premature ventricular beats," IEEE Trans. Biomed. Eng., vol. 52, no. 4, pp. 593-598, Apr. 2005.

[10] C. C. Lin, C. M. Chen, I. F. Yang, and T. F. Yang, "Automatic optimal order selection of parametric modeling for the evaluation of abnormal intra-QRS signals in signal-averaged electrocardiograms," Med. Biol. Eng. Comput., vol. 43, no. 2, pp. 218-224, Mar. 2005.

[11] C. C. Lin, "Enhancement of accuracy and reproducibility of parametric modeling for estimating abnormal intra-QRS potentials in signal-averaged electrocardiograms," Med. Eng. Phys., vol. 30, no. 7, pp. 834-842, Sep. 2008

[12] C. C. Lin, "Analysis of Unpredictable Intra-QRS Potentials in Signal-Averaged Electrocardiograms Using an Autoregressive Moving Average Prediction Model," Med. Eng. Phys., vol. 32, no. 2, pp. 136-144, Mar. 2010.

[13] M. H. Hayes, Statistical digital signal processing and modeling, Canada: John Wiley \& Sons, 1996, pp. 194, pp. 358-361.

[14] S. Haykin, Adaptive filter theory, 4th., New Jersey: Prentice-Hall, 2002, pp. 45-49.

[15] Y. H. Chan, "Biostatistics 102: quantitative data-parametric \& non-parametric tests," Med. J., Singapore, vol. 44, no. 8, pp. 391-396, Aug. 2003.

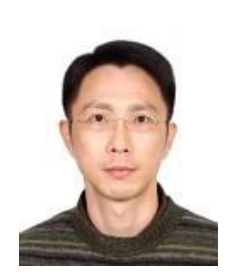

Chun-Cheng Lin was born in Taiwan, R.O.C., 1971. He received B.S.E.E., M.S.E.E., Ph. D. degrees all in electrical engineering from the National Taiwan University of Science and Technology, Taipei, Taiwan, in 1997, 1999, and 2005, respectively.

$\mathrm{He}$ is currently an associate professor in the Department of Electrical Engineering at National Chin-Yi University of Technology, Taiwan. He published over 50 articles in scientific journals and conferences, such as the Medical \& Biological Engineering \& Computing, the Medical Engineering \& Physics, and the Computers \& Mathematics with Applications. His major research interests include computerized electrocardiogram analysis, biomedical signal processing and modeling, and active noise control. 